\title{
Do Environmental Goals in Outdoor Education Reflect Client-needs or Outdoor Educator-needs?
}

\author{
Tony Pammer
}

\begin{abstract}
find myself increasingly questioning whether the collective thinking of Outdoor Educators is responsive to the needs of 'clients' or indicative of the views held by the individuals or collective who provide outdoor education.
\end{abstract}

In a recent informal survey at a Victorian Outdoor Education conference, completed by 50 delegates, by far the most prevalent view was that Outdoor Education existed to save or care for the environment. In response to the request "From your perspective, list the three most important aims of Outdoor Education..." there were four responses suggesting 'care/save the environment' for any one of the sum of all other responses. In response to question "In your opinion, how important will Outdoor Education be to the health and well being of a) individuals and communities comprising our society? and b) the environment in 2022? 56\% of respondents rated $\mathrm{OE}$ as of "great importance" to individuals and community, compared to $81 \%$ for the environment.

In travelling around Melbourne and Sydney talking to heads of independent schools, I am beginning to feel that it doesn't occur to leaders of these schools that outdoor education has much to offer in creating the outcomes they desire. In exploring this paradox, I sense that many people's understanding of the depth of outdoor education is limited.

On reflection, it strikes me that perhaps outdoor educators have spent a great deal of time telling each other what we think 'society' needs are (from outdoor education), and insufficient time asking 'society' what its needs are.

On balance I pondered the emerging possibility that Outdoor Educators wanted to 'save the environment' through outdoor education, whereas the schools I was talking to wanted and needed something else. This raises the question that my profession in fact seeks to impose its views on participants, without due consideration of what the perceived needs of these participants or their educational sponsors might be.
One of the many emerging questions is - do or should educators have clients? I have for a long time taken the view that they do - but am equally aware that many in our profession do not perceive students as clients. Does it follow that the representatives of students - parents, schools, school communities, youth agencies, government and non government agencies, are also not clients?

Should educators be market driven - or impose their views on the basis of "we know best"? Clearly there is a role for expert groups in society to be on the forward edge of thinking in their field, and to be trying to shape, or at least challenge ways of thinking. However, our profession needs to determine with its sponsors, agents \& clients, whether the collective feels social \& interpersonal issues impact on the environment and thus should be worked on before or at least in conjunction with. a preserve the environment theme.

My emerging conclusion is that our profession has already, or is at risk of, marginalising itself, by focussing too exclusively on a save the environment theme, when our clients have quite different, and very pressing needs. I feel our medium of learning is well suited to attend to developing these social/interpersonal needs, but we are quite failing to educate the community that outdoor education has developed far beyond the 1950's image of survival and 'toughen you up', that most people have of it. In my opinion it is high time for us to listen carefully to society's needs, match these to outdoor education's potential, and promote the benefits of outdoor education in relation to the perceived needs of our clients.

\section{About the Author:}

Tony has worked around the Outdoor Education traps for the last 20 years, generally causing trouble wherever he can. Currently employed by the Outdoor Education Group, he lives and works in Alexandra. Tony has dropped out of more university courses than most, and is generally renowned for his lack of education. Perhaps, however this gives him a more critical view of education and its role in Australian society. 